\title{
Desempenho acadêmico e autoconceito de escolares: contribuições do envolvimento paterno ${ }^{1}$
}

\author{
Children's academic performance and self-concept: \\ contributions of father involvement
}

\author{
Fabiana $\mathrm{Cl}^{2}$ \\ Elizabeth Joan BARHAM \\ Anne Marie Germaine Victorine FONTAINE ${ }^{3}$
}

\begin{abstract}
Resumo
Este estudo teve por objetivo examinar as relações entre três formas do envolvimento paterno (a comunicação entre pai e filho; a participação do pai nos cuidados com o filho; a participação do pai nas atividades escolares, culturais e de lazer do filho) e dois aspectos do desenvolvimento infantil (autoconceito e desempenho acadêmico) de crianças que iniciam as atividades escolares. Participaram deste estudo 97 casais e seus filhos matriculados na $1^{\mathrm{a}}$ ou $2^{\mathrm{a}}$ série do Ensino Fundamental. Para avaliar o envolvimento paterno a partir de duas perspectivas diferentes, tanto os pais quanto as mães responderam à Avaliação do bem-estar pessoal e familiar e do relacionamento pai-filho - Versão Paterna. Para avaliar o desempenho acadêmico e o autoconceito, foram aplicados nas crianças o Teste de Desempenho Escolar e o Self-Description Questionnaire I. Observou-se que, quanto maior a frequência de comunicação entre pai e filho, a participação do pai nos cuidados com o filho e a participação do pai nas atividades escolares, culturais e de lazer do filho, maior o desempenho acadêmico e o autoconceito das crianças. Esses resultados são indicativos da importância do envolvimento paterno e apontam a necessidade de se realizarem intervenções educativas dirigidas aos homens.
\end{abstract}

Unitermos: Autoconceito. Comportamento paterno. Estudante. Rendimento escolar.

\begin{abstract}
The aim of this study was to investigate the relationships among three types of fathering involvement (father-child communication, fathers' participation in caring for their child and fathers' participation in their child's school, cultural and leisure activities) and two aspects of a child's development (academic performance and self-concept) among children in their first years of schooling. The participants were 97 fathers, mothers and their children, who were attending the first or second grade. To evaluate father involvement from two different perspectives, both the fathers and the mothers completed the Evaluation questionnaire about individual and family well-being and the parent-child relationship-Fathering Version. Children's academic performance and self-concept were evaluated using the School Performance Test and the Self-Description Questionnaire I, respectively. The greater the frequency of father-child communication, of the fathers' participation in caring for their children, and in their children's school, cultural and leisure activities, the stronger the children's academic performance and the more positive their self-concept. These results are indicative of the importance offathers'involvement and point out the need for educational interventions specifically formen.
\end{abstract}

Unitermos: Self-concept. Paternal behavior. Student. Academic achievement.

\section{Vे}

1 Artigo elaborado a partir da tese de F. CIA, intitulada "Um programa para aprimorar envolvimento paterno impactos no desenvolvimento dos filhos". Universidade Federal de São Carlos, 2009. Apoio: Fundação de Amparo à Pesquisa do Estado de São Paulo.

2 Universidade Federal de São Carlos, Programa de Pós-Graduação em Educação Especial, Departamento de Psicologia. Rod. Washington Luís, Km 235, 13565-905, São Carlos, SP, Brasil. Correspondência para/Correspondence to: F. CIA. E-mail: <fabianacia@hotmail.com>.

3 Universidade do Porto, Faculdade de Psicologia e de Ciências da Educação, Departamento de Educação. Porto, Portugal. 
As relações familiares refletem diretamente nas diferentes áreas do desenvolvimento infantil e na emocionalidade (nível de stress, satisfação com a vida, bem-estar, entre outros aspectos) dos cuidadores. A exposição da criança a práticas parentais pouco construtivas ou sua privação de envolvimento afetivo com pais e mães constitui um fator de risco para seu desenvolvimento, aumentando sua vulnerabilidade a eventos ameaçadores externos ao ambiente familiar (Pacheco Alvarenga, Reppold, Piccinini \& Hutz, 2005). Em contrapartida, pais socialmente habilidosos, que estabelecem um ambiente familiar acolhedor, organizando contextos favoráveis, são mecanismos de proteção diante de fatores ameaçadores (por exemplo, pressões de grupos desviantes e exposição à violência fora de casa) aos quais as crianças usualmente estão expostas (Aunola \& Nurmi, 2005; Barros, 2006; Z.A.P. Del Prette \& A. Del Prette, 2005). Os pais são modelos de comportamento e fonte de significações para os filhos. Eles também selecionam e constroem os ambientes dos filhos, de acordo com os seus princípios e valores. São os pais que executam e planejam as atividades dos filhos, a partir dos interesses deles, seus e de terceiros (Barros, 2006). Partindo desse pressuposto, neste estudo são investigados os comportamentos paternos (comunicação entre pais e filhos, participação dos pais nos cuidados com os filhos e participação dos pais nas atividades escolares, culturais e de lazer dos filhos) e sua relação com dois aspectos do desenvolvimento infantil: autoconceito e desempenho acadêmico.

O autoconceito diz respeito ao conhecimento de si mesmo, com base nas características ou atributos que cada qual utiliza para se descrever. O autoconceito da criança em idade pré-escolar é concreto, e as autopercepções estão ligadas principalmente a características visíveis (por exemplo, altura, força física e aparência). Na idade escolar, com o desenvolvimento cognitivo, esse autoconceito muda em várias dimensões: de simples e global para diferenciado e articulado, de arbitrário e mutante para coerente e estável, de concreto para abstrato, de absoluto para relativo, do "eu" público ou externo para o "eu" privado e interno. As mudanças do autoconceito, ao longo da idade escolar, ocorrem tanto nos aspectos estruturais - das primeiras conexões entre conteúdos às generalizações e à integração - quanto em seus conteúdos de utilizar a si mesmo como referência a se comparar com os outros (Palácios \& Hidalgo, 2004).

No período inicial de escolarização, crianças entre seis e doze anos passam por duas fases distintas. Entre os seis e oito anos, a criança faz discriminações cada vez mais finas para descrever a si mesma, na base de comparações com seu próprio desempenho em momentos anteriores, sendo utilizados contrastes do tipo tudo ou nada. O "eu" é a principal referência, com maior ênfase em conteúdos internos e psicológicos. Na segunda fase, entre oito e doze anos, as relações interpessoais e as comparações com outras crianças tornam-se predominantes (Palácios \& Hidalgo, 2004). O autoconceito é influenciado por vários fatores, dentre os quais a participação paterna, conforme apontam vários estudos internacionais (Dekovic \& Meuis, 1997; Hong \& Ho, 2005; Verschueren \& Marcoen, 1999).

Numa pesquisa realizada no contexto brasileiro, Cia e Barham (2005) investigaram a influência da qualidade do relacionamento com o pai sobre o autoconceito do filho. Foram estudados 58 homens e seus filhos em idade escolar e que viviam com ambos os pais biológicos, em famílias com baixo poder aquisitivo. A coleta de dados envolveu o preenchimento de questionários pelo pai e pela criança, com o acompanhamento e ajuda da pesquisadora. Os resultados demonstraram que, quanto maior a frequência de envolvimento paterno (comunicação entre pai e filho e participação do pai nos cuidados com o filho) e quanto maior o tempo que o pai passava com o filho, melhor o autoconceito acadêmico (derivado de pontuações nas áreas de matemática, leitura e assuntos escolares em geral), o autoconceito não acadêmico (derivado de pontuações nas áreas de habilidades físicas, aparência física, relacionamento com ambos os pais e relacionamento com os colegas) e o autoconceito geral dos filhos.

A produção de conhecimento sobre o autoconceito torna-se primordial, considerando-se que várias pesquisas apontam essa variável como um dos principais construtos que indicam a adequação do desenvolvimento afetivo-emocional da criança, e também como um preditor crítico do rendimento acadêmico. Crianças com baixo autoconceito tendem a obter baixo rendimento acadêmico, o que, por sua vez, incrementa a avaliação negativa de si mesmo, mantendo um círculo vicioso (Chapman, Tunmer \& Prochnow, 2000; 
Formiga, 2004; Guay, Marsh \& Boivin, 2003; Okano, Loureiro, Linhares \& Marturano, 2004; Stevanato, Loureiro, Linhares \& Marturano, 2003). Nesse sentido, o impacto do envolvimento paterno sobre o desempenho acadêmico do filho pode contribuir tanto para manter, como para interromper esse círculo.

Os seguintes comportamentos parentais são apontados na literatura como favorecedores para o bom desempenho acadêmico dos filhos: comunicação entre pais e filhos (Cia, D'Affonseca \& Barham, 2004; Cia, Pamplin \&Williams, 2008; Hong \& Ho, 2005), participação e envolvimento dos pais em atividades diárias dos filhos (Bacarji, Marturano \& Elias, 2005; Englund, Luckner, Whaley \& Egeland, 2004; Flouri \& Buchanan, 2003), suporte emocional (Amato \& Gilbreth, 1999; Bacarji et al., 2005), participação em reuniões escolares (Hill \& Taylor, 2004), auxílio nas tarefas de casa e acompanhamento do progresso escolar (Hill \& Taylor, 2004).

Com o intuito de ajudar os pais a desenvolverem comportamentos que facilitam a aprendizagem e a participarem da vida acadêmica dos filhos, Soares, Souza e Marinho (2004) oferecem 14 orientações: "tornar explícitos os direitos e deveres dos filhos", "estabelecer uma rotina organizada, "estabelecer limites", "supervisionar atividades", "dosar adequadamente proteção e incentivo à independência", "prover um ambiente com recursos e instrumentos para estudar", "estabelecer interações positivas", "demonstrar afeto", "ser modelo adequado de envolvimento com as atividades", "promover diálogo", "apresentar nível de exigência compatível com o desempenho da criança", "relacionar o teórico com a prática", "incentivar o brincar"e "interessar-se pela vida do filho". Essas dicas ilustram padrões de qualidade para guiar o envolvimento parental.

Perante as pesquisas que evidenciam a influência do envolvimento paterno sobre o desenvolvimento infantil, a importância da realização do presente estudo se dá por duas questões. Primeiramente, as crianças desta amostra estão em fase de transição. Para Aspesi, Dessen e Chagas (2005), as fases de transição são definidas como passagens de um estágio de desenvolvimento para o outro. O início em um estágio novo exige que a criança se envolva em um conjunto de tarefas novas, o que leva o indivíduo em desenvolvimento a refinar o seu repertório e a adquirir diferentes competências para executá-las, de forma que passa por um processo de transição. As relações interpessoais, em especial familiares, têm impactos significativos durante todo o ciclo de vida, mas principalmente nas fases de transição. Situações como falta de estabilidade nos modelos e papéis familiares, alto índice de stress familiar, baixo envolvimento parental, elos emocionais fracos entre pais e filhos, baixo provimento de ambientes estimuladores do desenvolvimento emocional, cognitivo e social, são exemplos de fatores de risco à transição adaptativa entre os diferentes estágios do desenvolvimento. Assim, este estudo permite conhecer os impactos da qualidade de diferentes tipos de envolvimento paterno sobre o desenvolvimento do filho, em um período crítico do desenvolvimento infantil.

Como segundo motivo, verifica-se o quanto o envolvimento paterno positivo pode influenciar o desempenho acadêmico do filho, agindo como um fator de proteção sobre os possíveis fatores de risco e de maximização do desenvolvimento infantil, assim como contribuindo nas futuras etapas desenvolvimentais. Perante a escassez de estudos focalizando especificamente o envolvimento paterno (o que o pai faz, e não seu estilo parental), este estudo teve por objetivo examinar a relação entre três facetas do comportamento paterno (comunicação entre pai e filho, participação do pai nos cuidados com o filho e participação do pai nas atividades escolares, culturais e de lazer do filho) e dois aspectos do desenvolvimento infantil: autoconceito e desempenho acadêmico de crianças em início de atividades escolares.

\section{Método}

\section{Participantes}

Esta pesquisa contou com a participação de 97 casais (pais e mães) com filhos na $1^{\text {a }}$ ou $2^{\text {a }}$ séries do Ensino Fundamental (a "primeira série" equivale ao atual "segundo ano"). A idade média dos pais era de 35 anos, variando entre 23 e 58 anos, enquanto a idade média das mães era de 32 anos, oscilando entre 20 e 55 anos. Os pais eram todos casados e possuíam, em média, dois filhos. Em relação à classe socioeconômica, 7,1\% das famílias pertenciam à classe D, 50,5\% à classe C, 35,4\% à classe B2 e 7,1\% à classe B1 (segundo o Critério Brasil, 2006, que tem por objetivo medir o poder aquisi- 
tivo do consumidor e classificar a população em sete classes socioeconômicas). Todos os pais e 97\% das mães exerciam algum tipo de atividade remunerada. Como critério para participar da pesquisa, os pais (homens) tinham que exercer atividade remunerada, estar casados ou viver como casados.

Também participaram deste estudo 99 crianças, com idade média de oito anos, variando entre seis e nove anos. Destas crianças, 49 em do sexo masculino e 50 do feminino, sendo que $21,2 \%$ estavam na 1 a série do Ensino Fundamental, e 78,8\% na 2a série.

\section{Procedimentos éticos}

O estudo foi aprovado pelo Comitê de Ética em Pesquisa com Seres Humanos da Universidade Federal de São Carlos, sob o Protocolo CAAE 0074.0.135.000-05, em 8 de agosto de 2005. Todos os participantes assinaram um Termo de Consentimento Livre e Esclarecido antes de sua inclusão na amostra.

A coleta de dados referentes aos participantes ocorreu em duas escolas municipais e em uma escola estadual do interior do estado de São Paulo.

\section{Instrumentos}

\section{Envolvimento paterno na perspectiva de pais e mães}

"Avaliação do bem-estar pessoal e familiar e do relacionamento pai-filho - Versão paterna" (Cia, 2005). Os pais e as mães responderam individualmente a este instrumento, de forma que ambos avaliaram o envolvimento paterno. $O$ instrumento inclui três escalas: (a) Escala de comunicação (verbal e não verbal) entre pai e filho - escala tipo Likert de 22 itens, com pontuação variando entre 0 (nunca), 12 (uma vez por mês), 52 (uma vez por semana), 130 (duas ou três vezes por semana) e 365 (uma vez por dia) ( $\alpha=0,97)$; (b) Escala de participação do pai nos cuidados com o filho - escala tipo Likert de 15 itens, com pontuação variando entre 1 (nenhuma participação) e 5 (muita participação) $(\alpha=0,92)$; e (c) Escala de participação do pai nas atividades escolares, culturais e de lazer do filho - escala tipo Likert de 19 itens, com a pontuação variando entre 0 (nunca), 12 (uma vez por mês), 52 (uma vez por semana), 130 (duas ou três vezes por semana) e 365, (uma vez por dia) $(\alpha=0,95)$. $\bigcirc \alpha$ se refere à consistência interna da escala, sendo que quanto mais próximo ao valor 1, maior é a consistência. Quando o valor do $\alpha$ é superior a 0,70, considera-se que a escala possui boa consistência interna (Cozby, 2006).

\section{Medidas de aspectos do desenvolvimento das crianças}

Para avaliar o autoconceito das crianças, foi utilizado o Questionário para Avaliação do Autoconceito (Self-Description Questionnaire 1-SDQ1), elaborado por Marsh e Smith (1982), validado na Inglaterra e na Austrália, e adaptado para o contexto brasileiro por Garcia e De Rose (2000). O questionário é composto por 76 itens distribuídos em oito escalas, sete das quais apresentam índices de boa consistência interna (Habilidades Físicas - $\alpha=0,89$; Aparência Física - $\alpha=0,92$; Relacionamento com os Colegas - $\alpha=0,91$; Relacionamento com os Pais - $\alpha=0,90$; Leitura - $\alpha=0,93$; Matemática - $\alpha=0,51$; Assuntos Escolares em Geral - $\alpha=0,71$; e Autoconceito Geral - $\alpha=0,94)$. As oito escalas são divididas em duas categorias: autoconceito não acadêmico $(\alpha=0,96)$ e autoconceito acadêmico $(\alpha=0,94)$. Para preencher o questionário, as crianças respondem a cada afirmação usando uma escala de pontuação variando entre 1 (sempre falso) e 5 (sempre verdadeiro). A análise de consistência interna do instrumento como um todo apresentou um $\alpha=0,98$.

Para obter um índice do desempenho escolar das crianças, foi utilizado o Teste de Desempenho Escolar (TDE) (Stein, 1994), que é um instrumento com propriedades psicométricas adequadas (apresentando boa consistência interna para esta amostra - $\alpha=0,79)$ que avalia as capacidades fundamentais para o desempenho escolar. O teste foi elaborado para a avaliação de escolares de $1^{\text {a a }} 6^{\mathrm{a}}$ série do Ensino Fundamental e é composto por três escalas: (a) escrita - envolve a escrita do nome próprio e de 34 palavras isoladas, apresentadas sob a forma de ditado; (b) aritmética - requer a solução oral de três problemas e cálculos de 35 operações aritméticas por escrito; e (c) leitura - requer o conhecimento de 70 palavras isoladas do contexto.

\section{Procedimentos}

Após o consentimento dos pais e das mães, concordando em participar e autorizando a avaliação 
dos filhos, a primeira autora entrou em contato com os eles, para agendar um horário a fim de preencher o instrumento Avaliação do bem-estar pessoal e familiar e do relacionamento pai-filho - Versão paterna (tempo de aplicação - 40 minutos). O preenchimento do instrumento ocorreu em forma de entrevista com os pais; a pesquisadora lia cada item em voz alta e o participante respondia. As entrevistas ocorreram em uma sala de aula vazia, localizada nas dependências da própria escola.

Posteriormente, foram aplicados o SDQ1 (tempo de aplicação - 30 minutos) e o TDE (tempo de aplicação - 45 minutos). Primeiramente foi aplicado o SDQ1, em grupos de cinco crianças, para que elas se sentissem mais confiantes e menos ansiosas, e para que a coleta de dados ocorresse em um clima agradável e acolhedor. A pesquisadora lia cada um dos itens em voz alta e esperava as crianças responderem. Em seguida, era aplicado o TDE, começando com a escala de escrita (aplicada em grupo), seguida de avaliações individuais em aritmética e leitura. A aplicação dos testes seguiu as instruções no manual do instrumento, sendo sempre realizada em uma sala de aula vazia.

Os dados quantitativos, obtidos por meio do instrumento Avaliação do Bem-Estar Pessoal e Familiar e do relacionamento pai-filho - Versão paterna, foram analisados em termos descritivos, com medidas de tendência central e dispersão. A pontuação dos dados obtidos no SDQ1 e TDE foi realizada com base nos procedimentos apresentados no manual de cada teste.

Para correlacionar as medidas do relacionamento pai-filho, do desenvolvimento socioemocional e do desempenho acadêmico das crianças, foi necessário combinar os escores das crianças da $1^{\mathrm{a}}$ e da $2^{\mathrm{a}}$ série do Ensino Fundamental no TDE. Sempre que é preciso combinar dados de dois grupos independentes, com distribuições normais, mas médias diferentes, pode-se subtrair ou somar um valor fixo a todos os escores de um dos grupos, para transpor a média para o mesmo valor do segundo grupo, sem afetar a forma da distribuição dos escores do primeiro grupo (Hays, 1981). Sendo assim, optou-se por transformar os escores das crianças da $1^{\text {a }}$ série para se obter uma distribuição equivalente aos das crianças da $2^{a}$ série. Para tanto, foram acrescentados cinco pontos dos escores de cada criança da 1a série em aritmética, oito pontos em escrita, quatro pontos em leitura e, portanto, 17 pontos no escore total. Os valores fixos foram calculados por série, observando-se as diferenças nas médias normativas para cada subteste.

A relação entre frequência do envolvimento paterno, desempenho acadêmico e autoconceito das crianças foi verificada por meio do teste de correlação de Pearson. Para comparar a avaliação dos pais e das mães quanto ao envolvimento paterno, foi usado o Teste $t$.

\section{Resultados}

Na Tabela 1, apresentam-se os resultados dos valores médios e de dispersão obtidos pelos pais e pelas mães nos conjuntos de itens relacionados às três escalas de envolvimento paterno. Por meio do Teste t, verificou-se que não existiam diferenças estatisticamente significativas entre a frequência média de envolvimento paterno, segundo os pais e as mães, nas três áreas avaliadas: a) comunicação entre pais e filho $-M_{\text {pais }}=136,3, D P=87,5$;

Tabela 1. Medidas de tendência central e dispersão das escalas de envolvimento paterno, Universidade Federal de São Carlos. São Carlos (SP), 2006.

\begin{tabular}{|c|c|c|c|c|}
\hline Escalas & Média & DP & Valores mínimos & Valores máximos \\
\hline \multicolumn{5}{|l|}{ Opinião dos pais e mães } \\
\hline $\begin{array}{l}\text { Comunicação (verbal e não verbal) entre pais e filhos: } \\
0=\text { nunca a } 365=\text { todos os dias }\end{array}$ & 140,6 & 86,5 & 0 & 365 \\
\hline $\begin{array}{l}\text { Participação dos pais nos cuidados com os filhos: } \\
1=\text { pouca participação a } 5=\text { muita participação }\end{array}$ & 3,0 & 0,95 & 1 & 5 \\
\hline $\begin{array}{l}\text { Participação dos pais nas atividades escolares, culturais e de lazer com os filhos: } \\
0=\text { nunca a } 365=\text { todos os dias }\end{array}$ & 121,0 & 88,9 & 0 & 365 \\
\hline
\end{tabular}

DP: Desvio-Padrão. 
$\left.M_{\text {mães }}=144,9, D P=95,5 ; b\right)$ participação dos pais nos cuidados com os filhos $-M_{\text {pais }}=3,01, D P=0,99 ; M_{\text {mäes }}=3,05$, $\mathrm{DP}=0,97$; c) participação dos pais nas atividades escolares, culturais e de lazer dos filhos $-M_{\text {pais }}=121,7, D P=94,2$; $M_{\text {mäes }}=120,4, D P=90,1$.

Além disso, foi realizado o teste de correlação de Pearson. Verificou-se uma alta correlação entre os escores médios, atribuídos pelos pais e pelas mães aos três tipos de envolvimento paterno (comunicação entre pai e filho - $r=0,784$ e $p<0,001$; participação do pai nos cuidados com o filho - $r=0,882$ e $p<0,001$; participação do pai nas atividades escolares, culturais e de lazer dos filhos - $r=0,862$ e $p<0,001$ ). Assim, optou-se por calcular a pontuação média nessas escala, juntando as respostas dos pais e das mães, sendo apresentada apenas uma medida para cada escala. Cada pai recebeu uma pontuação média em cada escala, somando os escores do pai e da mãe, e depois dividindo por dois.

De modo geral, nas três escalas, segundo a opinião dos pais e das mães, os pais apresentaram uma frequência mediana de envolvimento (comunicação entre pais e filho - $M=140,6, D P=86,5$; participação dos pais nos cuidados com os filhos - $M=3,00, D P=0,95$; participação dos pais nas atividades escolares, culturais e de lazer dos filhos $-\mathrm{M}=121,0, \mathrm{DP}=88,9$ ).

Na Tabela 2, apresenta-se a avaliação do desempenho acadêmico das crianças, aferido peloTDE.
A pontuação média para o grupo de crianças que participaram deste estudo indica um desempenho acadêmico igual ao esperado em aritmética, escrita, leitura e no escore total do TDE (Aritmética $=12,1$; Escrita = 25,9; Leitura =60,6; Escore total =98,6), de acordo com as normas do instrumento (Stein, 1994). Na Tabela 3, apresentam-se os valores médios obtidos pelas crianças em relação a seu autoconceito.

Segundo a amostra de referência, as crianças, em média, apresentaram um autoconceito satisfatório, ou seja, apresentaram um valor mediano, de acordo com as normas de referência do teste (Garcia \& De Rose, 2000).

Na Tabela 4, são apresentadas as correlações significativas entre os escores obtidos nos subtestes do Self-Description Questionnaire I-SDQ1 e do TDE, e as escalas que avaliaram o envolvimento paterno.

Os escores médios nas escalas usadas para avaliar o envolvimento paterno (comunicação entre pais e filhos, participação dos pais nos cuidados com os filhos e participação dos pais nas atividades escolares, culturais e de lazer dos filhos) correlacionaram-se significativamente com o desempenho acadêmico global das crianças (escore total no TDE) e com pelo menos duas dimensões mais específicas do desempenho acadêmico, segundo o TDE, como mostra a Tabela 4. Além disso, as escalas de comunicação entre

Tabela 2. Desempenho acadêmico das crianças - TDE, Universidade Federal de São Carlos. São Carlos (SP), 2006.

\begin{tabular}{lcccc}
\hline Subtestes - TDE & Média & DP & Escore médio (2a série) & Pontuação máxima \\
\hline Aritmética & 12,1 & 3,39 & $10-13$ & 38 \\
Escrita & 25,9 & 7,44 & $20-26$ & 35 \\
Leitura & 60,6 & 13,90 & $58-66$ & 70 \\
Escore total & 98,6 & 21,70 & $87-105$ & 143 \\
\hline
\end{tabular}

TDE: Teste de Desempenho Escolar; DP: Desvio-Padrão.

Tabela 3. Autoconceito das crianças, Universidade Federal de São Carlos. São Carlos (SP), 2006.

\begin{tabular}{|c|c|c|c|c|c|}
\hline \multirow{3}{*}{$\begin{array}{l}\stackrel{\pi}{0} \\
\stackrel{0}{D} \\
\stackrel{D}{+} \\
\cong\end{array}$} & Autoconceito & Média & DP & Pontuação mínima & Pontuação máxima \\
\hline & $\begin{array}{l}\text { Autoconceito não acadêmico (média: habilidades físicas, aparência } \\
\text { física, relacionamento com os pais e com os colegas) }\end{array}$ & 28,8 & 7,30 & 10,50 & 39,5 \\
\hline & $\begin{array}{l}\text { Autoconceito acadêmico (média: matemática, leitura e assuntos } \\
\text { escolares em geral) }\end{array}$ & 30,2 & 8,95 & 9,33 & 40,0 \\
\hline- & Autoconceito total (média de todas as habilidades) & 29,5 & 7,86 & 9,92 & 40,0 \\
\hline
\end{tabular}

466

A pontuação máxima para cada aspecto medida no Self-Description Questionaire é 40. DP: Desvio-Padrão. 
Tabela 4. Correlações (Pearson) significativas entre o desempenho acadêmico das crianças e o envolvimento. Universidade Federal de São Carlos. São Carlos (SP), 2006.

\begin{tabular}{|c|c|c|c|}
\hline $\begin{array}{l}\text { Variáveis do desempenho acadêmico e do } \\
\text { autoconhecimento }\end{array}$ & $\begin{array}{l}\text { Comunicação } \\
\text { entre pai e filho }\end{array}$ & $\begin{array}{l}\text { Participação do pai nos } \\
\text { cuidados com o filho }\end{array}$ & $\begin{array}{l}\text { Participação do pai nas } \\
\text { atividades escolares, } \\
\text { culturais e de lazer do filho }\end{array}$ \\
\hline \multicolumn{4}{|l|}{ Desempenho acadêmico - TDE } \\
\hline Aritmética & $0,263^{* *}$ & ns & $0,219^{*}$ \\
\hline Escrita & $0,269^{* *}$ & $0,239^{*}$ & $0,257^{*}$ \\
\hline Leitura & ns & $0,207^{*}$ & NS \\
\hline Escore total & $0,257^{*}$ & $0,246^{*}$ & $0,256^{*}$ \\
\hline \multicolumn{4}{|l|}{ Autoconceito - SDQ } \\
\hline Habilidades Físicas & $0,392^{* * *}$ & $0,345^{* * *}$ & $0,412^{* * *}$ \\
\hline Aparência Física & $0,432^{* * *}$ & $0,339^{* *}$ & $0,401^{* * *}$ \\
\hline Relacionamento com os colegas & $0,388^{* * *}$ & $0,264^{* *}$ & $0,363^{* * *}$ \\
\hline Relacionamento com os pais & $0,299^{* *}$ & ns & $0,257^{*}$ \\
\hline Leitura & $0,316^{* *}$ & ns & $0,281^{* *}$ \\
\hline Matemática & $0,323^{* *}$ & $0,218^{*}$ & $0,298^{* *}$ \\
\hline Assuntos escolares em geral & $0,333^{* *}$ & $0,198^{*}$ & $0,300^{* *}$ \\
\hline Autoconceito geral & $0,422^{* * *}$ & $0,311^{* *}$ & $0,410^{* * *}$ \\
\hline $\begin{array}{l}\text { Autoconceito não acadêmico (média: habilidades físicas, } \\
\text { aparência física, relacionamento com os pais e com os } \\
\text { colegas) }\end{array}$ & $0,416^{* * *}$ & $0,313^{* *}$ & $0,394^{* * *}$ \\
\hline $\begin{array}{l}\text { Autoconceito acadêmico (média: matemática, leitura e } \\
\text { assuntos escolares em geral) }\end{array}$ & $0,333^{* *}$ & $0,198^{*}$ & $0,301^{* *}$ \\
\hline Autoconceito total (média de todas as habilidades) & $0,371^{* * *}$ & $0,246^{*}$ & $0,347^{* * *}$ \\
\hline
\end{tabular}

Nota: ${ }^{*} p<0,05 ;{ }^{* *} p<0,01 ;{ }^{* * *} p<0,001 ;$ ns = não apresenta diferenças estatisticamente significativas.

TDE: Teste de Desempenho Escolar; SDQ: Self-Description Questionnaire.

pai e filho e a de participação do pai nas atividades escolares, culturais e de lazer do filho, estavam positivamente correlacionadas com o autoconceito não acadêmico, acadêmico e total das crianças, obtendo correlações de ordem similar (baixo a moderado).

\section{Discussão}

De modo geral, as crianças apresentaram autoconceito e desempenho acadêmico igual ao esperado. Manter-se dentro dos padrões populacionais para sua faixa etária é importante, considerando principalmente que as crianças deste estudo estão em fase de transição, entre a pré-escola e o ensino fundamental, em que habilidades mais complexas Ihes são exigidas (Aspesi et al., 2005; Marturano, 2004). Por exemplo, as crianças precisam se adaptar às novas regras da escola, recebendo menor suporte de adultos para realizar atividades, quando comparadas às crianças na pré-escola (Marturano, 2004). Assim, o desenvolvimento emocional da criança (como por exemplo, ter um bom autoconceito) e seu desempenho acadêmico (como por exemplo, capacidade de acompanhar as tarefas solicitadas pelo professor em sala de aula) agem como fatores de proteção para seu desenvolvimento durante essa fase de transição (Moreno, 2004).

Além dos indicadores do desenvolvimento normativo das crianças, os relatos dos participantes adultos indicaram bom envolvimento dos pais, mantendo um padrão cotidiano de comunicação bidirecional, assumindo cuidados do dia a dia e acompanhando as diferentes atividades dos filhos. Tais aspectos são importantes porque, quando a criança ingressa na escola, os padrões de interação social entre pai e filho também se modificam. Os pais tornam-se mais exigentes com os filhos, tanto para a execução das tarefas domésticas, quanto para as tarefas acadêmicas. Ao mesmo tempo, usam novos meios de disciplina com os filhos, deixando de usar métodos de controle direto, que são substituídos por instruções diretivas sobre o comportamento desejado, utilizando o humor, o apelo à autoestima e o despertar da culpa (M. Cole \& S.R. Cole, 2004). Essa mudança ocorre em virtude do desenvolvimento psicossocial da criança, em conjunto com o fato de que a família tem 
que se adaptar aos padrões de comportamento infantil e às práticas de disciplina vigentes no meio escolar. Essas forças desencadeiam uma reorganização das vivências familiares. As diferenças nas práticas e normas que existem em cada família evidenciam-se nos comportamentos do grupo de colegas de escola. Os pais precisam ajudar seu filhos a compreender as consequências a curto e a longo prazo de cada conduta e a motivá-los a manter os comportamentos que consideram mais adaptados à realidade de sua vida. Diante dessas adaptações e da necessidade de aprender a lidar com novas demandas sociais e cognitivas, a interação entre pais e filhos torna-se muito significativa. Quanto maior o apoio e a qualidade das interações que ocorrem na convivência entre pais e filhos, mais rapidamente a criança aprenderá a lidar com as demandas e, portanto, menos estressante será essa fase para ela e familiares (P.A. Cowan \& C.P. Cowan, 2003).

Em relação às correlações entre as escalas usadas para avaliar o envolvimento paterno e os subtestes do TDE, nota-se que há uma variedade de comportamentos paternos que influenciam no bom desempenho escolar dos filhos. Por exemplo, a frequência das conversas entre pais e filhos, da participação daqueles na rotina doméstica e seu acompanhamento das demais atividades destes, favorecem o desempenho acadêmico das crianças em relação à escrita e à pontuação total do TDE. Esses dados vêm corroborar os resultados de outros estudos sobre os benefícios do envolvimento paterno para o desempenho acadêmico dos filhos (Bacarji et al., 2002; Cia et al., 2004; Cia et al., 2008; Duch, 2005; Flouri \& Buchanan, 2003; Hong \&Ho, 2005; Hubner, 2002; Morrison Brown, D'Incau, O'Farrell \& Furlong, 2006; Overstreet, Devine, Bevans \& Efreom, 2005). Trivellato-Ferreira e Marturano (2008), em um estudo brasileiro com crianças que estavam ingressando no Ensino Fundamental, verificaram que os recursos do ambiente familiar e as práticas parentais positivas foram preditores do desempenho acadêmico, do ajustamento escolar e da competência social das crianças. No mesmo sentido, Soares et al. (2004) apresentam evidências mostrando que comportamentos parentais de promover diálogos e se interessar pelas atividades do filho podem auxiliar em seu desempenho acadêmico.

A comunicação entre pai e filho e a participação do pai nas atividades escolares, culturais e de lazer dos filhos também estavam positivamente correlacionadas com o desempenho acadêmico das crianças em aritmética. O papel paterno centra-se no desenvolvimento de atividades lúdicas e de lazer com os filhos, favorecendo as interações verbais entre eles. Considerando que, para Piaget, os três fatores que explicam o desenvolvimento são a maturação, a experiência com os objetos e a experiência com as pessoas, o envolvimento paterno permite que os filhos sejam estimulados em diversos tipos de operações descritas como essenciais para a construção do pensamento operacional concreto (classificação, seriação, conservação numérica, mediação, assim como a noção de reversibilidade, descentração e conservação), o que por sua vez, torna-se a base para as operações lógico-matemáticas (Martí, 2004).

Todas as facetas do autoconceito também estavam positivamente correlacionadas com as escalas de comunicação entre pai e filho e de participação do pai nas atividades escolares, culturais e de lazer do filho. Esses dados corroboram outras pesquisas que apontam que a qualidade do relacionamento entre pai e filho favorece o autoconceito filial (Cia \& Barham, 2005; Dekovic \&Meuis, 1997; Verschueren \& Marcoen, 1999).

Ressalta-se o alto envolvimento familiar dos pais desta amostra. Os comportamentos relatados e a frequência com a qual são praticados são indícios de que eles atribuem importância para a interação familiar, para além das atividades profissionais. Ou seja, provavelmente conseguem equilibrar as demandas profissionais e familiares, demonstrando padrões esperados de uma nova paternidade, em que os pais não estão sendo apenas os provedores financeiros da família, mas estão participando junto com suas esposas nos cuidados e na educação dos filhos (Brandth \& Kvande, 2002; Cabrera, Tames-LeMonda, Bradley, Hofferth \& Lamb, 2000; Cia, Pereira, Z. A. P. Del Prette \& A. Del Prette, 2006; Matta \& Knudson-Martin, 2006; Tiedje, 2004; Yeung, Sandbergm, Davis-Kean \& Hofferth, 2001).

No geral, essa nova divisão de tarefas, com os cônjuges comprometidos com a responsabilidade conjunta em relação às atribuições familiares, parece resultar em melhor relacionamento entre ambos e destes com os filhos (Cia et al., 2006). Essa mudança cultural pode reverter em uma melhor qualidade da interação entre pais e filhos, favorecendo o desenvolvimento infantil (Bolsoni-Silva \& Del Prette, 2002; Overstreet et al., 2005; Willman, Phillips, Dunphy-Lelli \& Lanlonde, 2004). 


\section{Considerações Finais}

Os resultados do presente estudo apontam indicadores de comportamento paterno que influenciam o desempenho acadêmico dos filhos. De modo geral, quanto maior a frequência da comunicação entre pai e filho, dos cuidados com o filho e da participação do pai nas atividades escolares, culturais e de lazer do filho, maior o autoconceito e o desempenho acadêmico das crianças. Programas educacionais dirigidos para o pai, visando aprimorar o envolvimento e as práticas parentais, poderiam promover um desenvolvimento mais saudável dos filhos e, por consequência, diminuir o número de crianças classificadas com necessidades educacionais especiais, por apresentar baixo desempenho acadêmico ou problemas socioemocionais.

Deve-se ressaltar que esta pesquisa caracteriza-se como estudo correlacional, não sendo possível estabelecer relação causal entre as variáveis investigadas. Além disso, o instrumento utilizado "Avaliação do bem-estar pessoal e familiar e do relacionamento pai-filho - Versão Paterna" ainda não foi validado, embora a realização de análises estatísticas tenha sugerido sua validade interna. Estudos longitudinais seriam indicados para monitorar a influência do envolvimento parental sobre o desenvolvimento social dos filhos, ao longo do período do desenvolvimento infantil.

\section{Referências}

Amato, P. R., \& Gilbreth, J. G. (1999). Nonresident fathers and children's well-being: a meta-analyses. Journal of Marriage and the Family, 61 (1), 557-573.

Aspesi, C. C., Dessen, M. A., \& Chagas, J. F. (2005). A ciência do desenvolvimento humano: uma perspectiva interdisciplinar. In M. A. Dessen \& A. L. C. Costa (Orgs.), A ciência do desenvolvimento humano: tendências atuais $e$ perspectivas futuras (pp.19-36). Porto Alegre: Artmed.

Aunola, K., \& Nurmi, J. E. (2005). The role of parenting styles in children's problem behavior. Child Development, 76 (6), 1144-1159.

Bacarji, K. M. G., Marturano, E. M., \& Elias, E. C. S. (2005). Suporte parental: um estudo sobre crianças com queixas escolares. Psicologia em Estudo, 10 (1), 107-115.

Barros, L. (2006). Significações parentais: desenvolvimento e intervenção. Revista Portuguesa de Psicologia, 39, 65-89.

Bolsoni-Silva, A. T., \& Del Prette, A. (2002). O que os pais falam sobre suas habilidades sociais e de seus filhos? Argumento, 3 (7), 71-86.

Brandth, B., \& Kvande, E. (2002). Reflexive fathers: negotiating parental leave and working life. Gender, Work and Organization, 9 (2), 186-203.
Cabrera, N. J., Tames-LeMonda, C., Bradley, R. H., Hofferth, S., \& Lamb, M. E. (2000). Fatherhood in the twenty first century. Child Development, 71 (1), 127-136.

Chapman, J. W., Tunmer, W. E., \& Prochnow, J. E. (2000). Early reading-related skills and performance, reading self-concept, and the development of academic self-concept: A longitudinal study. Journal of Educational Psychology, 92 (4), 703-708.

Cia, F. (2005). O impacto do turno de trabalho do pai no desempenho acadêmico e no autoconceito de crianças escolares. Dissertação de mestrado não-publicada, Programa de Pós-Graduação em Educação Especial, Universidade Federal de São Carlos.

Cia, F., \& Barham, E. J. (2005). A relação entre o turno de trabalho do pai e o autoconceito do filho. Psico, 36 (1), 29-35.

Cia, F., D'Affonseca, S. M., \& Barham, E. J. (2004). A relação entre envolvimento paterno e desempenho acadêmico dos filhos. Paidéia: Cadernos de Psicologia e Educação, 14 (29), 277-286.

Cia, F., Pamplin, R. C. O., \& Williams, L. C. A. (2008). O impacto do envolvimento parental no desempenho acadêmico de crianças escolares. Psicologia em Estudo, 13 (2), 251-260.

Cia, F., Pereira, C. S., Del Prette, Z. A. P. \& Del Prette, A. (2006). Habilidades sociais parentais e o relacionamento entre pais e filhos. Psicologia em Estudo, 11 (1), 73-81.

Cole, M., \& Cole, S. R. (2004). Desenvolvimento social na segunda infância. In M. Cole \& S. R. Cole (Orgs.), O desenvolvimento da criança e do adolescente (pp.570-615). Porto Alegre: Artmed.

Cowan, P. A., \& Cowan, C. P. (2003). Normative family transitions, normal family processes, and healthy child development. In F. Walsh (Org.), Normal family processes: growing diversity and complexity (pp.424-459). New York: The Guilford Press.

Cozby, P. C. (2006). Métodos de pesquisa em ciências do comportamento. São Paulo: Atlas.

Critério Brasil. (2006). Associação Brasileira de Empresas de Pesquisa. Recuperado em janeiro 5, 2007, disponível em <http://www.abep.org>.

Dekovic, M., \& Meuis, W. (1997). Peer relations in adolescence: effects of parenting on adolescents' self-concept. Journal of Adolescence, 97 (20), 1163-1176.

Del Prette, Z. A. P., \& Del Prette, A. (2005). Psicologia das habilidades sociais na infância: teoria e prática. Petrópolis: Vozes.

Duch, H. (2005). Redefining parent involvement in Head Start: a two-generation approach. Early Child Development and Care, 175 (1), 23-35.

Englund, M. M., Luckner, A. E., Whaley, G. J. L., \& Egeland, B. (2004). Children's achievement in early elementary school: longitudinal effects of parental involvement, expectations, and quality of assistence. Jounal of Educacional Psychology, 96 (4), 723-730.

Flouri, E., \& Buchanan, A. (2003). The role of father involvement in children's later mental health. Journal of Adolescence, 26 (1), 63-78. 
Formiga, N. S. (2004). O tipo de orientação cultural e sua influência sobre os indicadores do rendimento escolar. Psicologia: Teoria e Prática, 6 (1), 13-29.

Garcia, S. C., \& De Rose, T. M. S. (2000). Autoconceito e desempenho escolar. Monografia não-publicada, Curso de Psicologia, Universidade Federal de São Carlos.

Guay, F., Marsh, H. W., \& Boivin, M. (2003). Academic selfconcept and academic achievement: developmental perspectives on their causal ordering. Journal of Educational Psychology, 95 (1), 124-136.

Hays, W. L. (1981). Statistics. New York: Holt Reinhart \& Winston.

Hill, N. E, Castellino, D. R., Lansford, J. E., Nowlin, P., Dodge, K. A., Bates, J. E., et al. (2004). Parent academic involvement as related to school behavior, achievement and aspirations: demographic variations across adolescence. Child Development, 75 (5), 1491-1509.

Hill, N. E., \& Taylor, L. C. (2004). Parental school involvement and children's academic achievement. Current Directions in Psychological Science, 13 (4), 161-164.

Hong, S., \& Ho, H. (2005). Direct and indirect longitudinal effects of parental involvement on student achievement: second-orde latent growth modeling across ethnic groups. Journal of Educational Psychology, 97 (1), 32-42.

Hubner, M. M. C. (2002). A importância da participação dos pais no desempenho escolar dos filhos: ajudando sem atrapalhar. In M. Z. S. Brandão, F. C. S. Conte \& S. M. B. Mezzaroba (Orgs.), Comportamento humano: tudo ou (quase tudo) que você precisa saber para viver melhor (pp.139-146). Santo André: ESETec.

Marsh, H. W., \& Smith, I. D. (1982). Multitrait-multimethod analyses of two self-concept instruments. Journal of Educational Psychology, 74 (3), 430-440.

Martí, E. (2004). Processos cognitivos básicos e desenvolvimento intelectual entre os seis anos e a adolescência. In C. Coll, A. Marchesi \& J. Palácios (Orgs.), Desenvolvimento psicológico e educação (pp.233-251). Porto Alegre: Artmed.

Marturano, E. M. (2004). Fatores de risco e proteção no desenvolvimento sócio-emocional de crianças com dificuldades de aprendizagem. In E. G. Mendes, M. A. Almeida \& L. C. A. Williams (Orgs.), Avanços recentes em Educação Especial (pp.159-165). São Carlos: EDUFSCar.

Matta, D. S., \& Knudson-Martin, C. (2006). Father responsivity: couple processes and the coconstruction of fatherhood. Family Process, 45 (1), 19-37.

Moreno, M. D. C. (2004). Desenvolvimento e conduta social dos seis anos até a adolescência. In C. Coll, A. Marchesi \& J. Palácios (Orgs.), Desenvolvimento psicológico eeducação: psicologia evolutiva (pp.287-305). Porto Alegre: Artmed.

Morrison, G. M., Brown, M., D'Incau, B., O'Farrell, S. L., \& Furlong, M. J. (2006). Understanding resilience in educational trajectories: implications for protective possibilities. Psychology in the Schools, 43 (1), 19-31.

Okano, C. B., Loureiro, S. R., Linhares, M. B. M., \& Marturano, E. M. (2004). Crianças com dificuldades escolares atendidas em programa de suporte psicopedagógico na escola: avaliação do autoconceito. Psicologia: Reflexão e Crítica, 17 (1), 121-128

Overstreet, S., Devine, J., Bevans, K., \& Efreom, Y. (2005). Predicting parental involvement in children's schooling within an economically disadvantaged African American sample. Psychology in the Schools, 42 (1), 101-111.

Pacheco, J., Alvarenga, P., Reppold, C., Piccinini, C. A., \& Hutz, C. S. (2005). Estabilidade do comportamento antisocial na transição da infância para a adolescência: uma perspectiva desenvolvimentista. Psicologia: Reflexão e Crítica, 18 (1), 55-61

Palácios, J., \& Hidalgo, V. (2004). Desenvolvimento da personalidade dos seis até a adolescência. In C. Coll, A. Marchesi \& J. Palácios (Orgs.), Desenvolvimento psicológico eeducação:psicologia evolutiva (pp.252-267). Porto Alegre: Artmed.

Soares, M. R. Z., Souza, S. R., \& Marinho, M. L. (2004). Envolvimento dos pais: incentivo à habilidade de estudo em crianças. Estudos de Psicologia (Campinas), 21 (3), 253-260. doi: 10.1590/S0103-166X2004000300009.

Stein, L. M. (1994). Teste de desempenho escolar. São Paulo: Casa do Psicólogo.

Stevanato, I. S, Loureiro, S. R., Linhares, M. B. M., \& Marturano, E. M. (2003). Autoconceito de crianças com dificuldades de aprendizagem e problemas de comportamento. Psicologia em Estudo, 8 (1), 67-76.

Tiedje, L. B. (2004). Process of change in work/home incompatibilities employed mothers. Journal of Social Issues, 60 (4), 787-800.

Trivellato-Ferreira, M. C., \& Marturano, E. M. (2008). Recursos da criança, da família e da escola predizem competência na transição da 1 a série. Interamerican Journal of Psychology, 42 (3), 549-558.

Verschueren, K., \& Marcoen, A. (1999). Representation of self and socioemotional competence in kindergartners: differential and combined effects of attachment to mother and to father. Child Development, 70 (1), 183-201.

Willman, H. M., Phillips, A. T., Dunphy-Lelli, S., \& Lanlonde, N. (2004). Infant social attention predicts preschool social cognition. Developmental Science, 7 (3), 283-288.

Yeoung, W. J., Sandberg, J. F., Davis-Kean, P. E., \& Hoffert, S. L. (2001). Children's time with father in intact families. Journal of Marriage and Family, 63 (1), 136-154.

Recebido em: 30/1/2010

Versão final em: 24/2/2012

Aprovado em: 13/3/2012 